J. Austral. Math. Soc. Ser. B 29 (1987), 59-78

\title{
ON THE ANALYSIS OF JOINT PRODUCTION
}

\author{
J. E. WOODS
}

(Received 4 December 1984; revised 14 January 1986)

\begin{abstract}
It is well known that $n$-process, $n$-commodity (or square), models of productive singleproduct industries have positive solutions to their price and quantity systems if the rates of profit and growth lie in appropriate non-negative intervals. On the other hand, negative prices and quantities can occur in formal solutions of models of square, productive, multiple-product industries even when the rates of profit and growth are less than their respective maximum positive values. It is shown in this paper that these differences can be attributed to the presence in joint production of dominance, in either row or column versions. Results on positive solutions to the price (respectively, quantity) system are derived in terms of the absence of column (respectively, row) dominance of the net output matrix. As the concepts of row and column dominance are defined in terms of linear inequalities, the basic mathematical results to be applied are theorems of the alternative.
\end{abstract}

\section{Introduction}

1.1. The properties of economic models in which commodities are produced by means of themselves and labour in models of single-product industries are by now well known - see, for example, Part I of Sraffa [13], Pasinetti [12] or Burmeister and Dobell [2]. Less well known are the properties of models with multiple-product industries, a class which subsumes not only fixed capital and natural resources but also intrinsic joint production-see Part II of Sraffa [13]. The purpose of this paper is to attempt to rectify this omission by providing a unified account of a simple joint production model.

\footnotetext{
${ }^{1}$ Department of Economics, Queen Mary College, University of London, Mile End Road, London E1 4NS England

(O) Copyright Australian Mathematical Society 1987, Serial-fee code 0334-2700/87
} 
To be specific, we shall analyse an $n$-commodity, $n$-process model satisfying these assumptions:

(A1): each process exhibits constant returns to scale;

(A2): each process produces a positive net output of at least one commodity;

(A3): for each commodity, a positive net output is produced by at least one process;

(A4): there is only one non-produced input, homogeneous labour, which is essential to each process.

On the basis of (A1), it is possible to describe a typical process, the $j$ th, by the triple $\left\{A^{j}, \ell_{j}: B^{j}\right\}$, where $A^{j} \equiv\left[a_{i j}\right]$ is the column vector of produced inputs, $\ell_{j}$ the direct labour input, both into the $j$-th process, and $B^{j} \equiv\left[b_{i j}\right]$ is the column vector of commodity outputs from the $j$-th process, $i, j=1, \ldots, n$. The commodity input vectors, the direct labour input coefficients and commodity output vectors can be stacked to give the triple $\{A, \ell: B\}$, which completely describes the technology for the economy; obviously, $A \equiv\left[A^{J}\right], \ell \equiv\left[\ell_{j}\right], B \equiv\left[B^{J}\right], j=$ $1, \ldots, n$.

In examining the price system, our main concern will be with prices of production which support a uniform rate of profit. For the model outlined above, we seek a semipositive solution $(p, w, r)$ to:

$$
p B=(1+r) p A+w \ell,
$$

where $p \equiv\left[p_{1}\right]$ is the row vector of produced commodity prices, $i=1, \ldots, n, w$ and $r$ are the uniform rates of wages and profits respectively. If the wage rate is positive, we can divide through (1) by $w$ to obtain:

$$
\tilde{p} B=(1+r) \tilde{p} A+\ell,
$$

where $\tilde{p} \equiv p / w$ is the vector of labour-commanded prices. In addition to (1) and (2), we shall also consider:

$$
p B=p A(I+R)+w \ell
$$

and

$$
p B=(1+r) p A+\ell W .
$$

In (3), $R=\left[r_{i} \delta_{i j}\right]$, where $r_{i}$ is the rate of profit obtained by operating the $i$-th process; in (4), $W=\left[w_{l} \delta_{i}\right], w_{l}$ being the wage rate paid by the $i$-th process. So, in (3), there are profit rate differentials but a uniform wage, with the position reversed in (4). On the quantity side, we seek a semipositive solution $(x, g)$ to:

$$
B x=(1+g) A x+c,
$$

where $x \equiv\left[x_{i}\right]$ and $c \equiv\left[c_{i}\right]$ are the column, vectors of activity levels and computation respectively, $i=1, \ldots, n$, and $g$ is the uniform rate of growth. Clearly, (5) is dual to (2). 
For a model of single-product industries with circulating capital, $B=I$ in the equations above. The imposition of a productiveness condition such as:

$$
\exists x \geqslant 0 \text { such that } x>A x
$$

permits the simultaneous solution of both price and quantity systems, (12) and (5). On the basis of (6), it follows that there exists a positive maximum value of $r$, denoted by $r_{\max }$, such that:

(a) $w=w(r)$ is a decreasing differentiable function of $r$, with $w_{\max }=w(0)>0$ and $0=w\left(r_{\text {max }}\right)$;

(b) $p \geqslant 0$ for all $r \in\left[0, r_{\max }\right]$.

Furthermore, there exists a positive maximum value of $g$ denoted by $g_{\max }$, such that:

(c) $c=c(g)$ is a decreasing differentiable function of $g$, with $c_{\max }=c(0)>0$ and $0=c\left(g_{\max }\right)$;

(d) $x \geqslant 0_{n}$ for all $g \in\left[0, g_{\max }\right]$;

(e) $g_{\max }=r_{\max }{ }^{2}$

The imposition of a productiveness condition, analogous to (6), on the joint production model (1) and (5) does not yield results similar to (a)-(e), except in special circumstances. ${ }^{3}$ Our purpose being to consider a general, not a specific, $n$-process, $n$-commodity joint production model, it will be necessary to adopt an alternative approach based on the recognition that a multiple-product industries system is essentially different from a single-product industries system.

1.2. Consider first an $n$-process, $n$-commodity single-product industries model. As each process produces a net output of only one commodity, it follows that if there is, for example, a final demand for commodity $i$, process $i$ and all others directly and indirectly connected with it must be operated. This correspondence between commodities and processes is lost in a pure joint production model, which raises the possibility that a subset of processes taken together can produce more of each commodity than the complementary subset of processes. Such a possibility, which arises for an $n$-process, $n$-commodity model from the assumption of joint production, cannot occur in a single-product industries model unless there are more processes than commodities. In other words, it is possible that a subset of processes is in some sense more efficient than, or dominates, the complementary subset of processes. This notion of dominance, which is thus a

\footnotetext{
${ }^{2}$ For the derivation of (a)-(e), consult Pasinetti [12] or Burmeister [2], for example. These results can be obtained by employing either Perron-Frobenius Theory on semi-positive matrices, the theory of $M$-matrices or the notion of diagonal dominance: on the equivalence between these approaches, see Woods [17] or Berman and Plemmons [1].

${ }^{3}$ E.g. any hypothesis which implies $(B-A)^{-1} \geqslant[0]$. For (1) and (5) can then be effectively converted into a single-product industries model by the method of vertical integration: see Pasinetti [11].
} 
distinguishing feature between single- and multiple-product industries models, each having $n$ processes and $n$ commodities, will play a central role in the analysis below. Other references employing this concept include Dumenil and Levy [3], Filippini [4], Filippini and Filippini [5], [6], Fujimori [7] and Levy [9].

We distinguish between two classes of dominance-defined with respect to either columns or rows of a matrix - there being five types within each class.

Definition 1. The matrix $C$ exhibits column dominance of the first type with respect to $u$ (CD1) if $\exists a \geqq 0, b \geqq 0, a^{\prime} b=0$ such that:

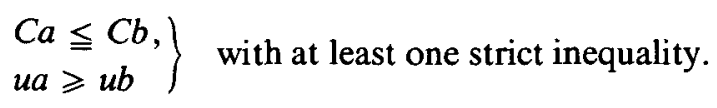

(6) can be rewritten as:

$$
\left.\begin{array}{c}
C(b-a) \geqq 0, \\
-u(b-a) \geqslant 0
\end{array}\right\} \quad \text { with at least one strict inequality }
$$

or

$$
\left[\begin{array}{c}
C \\
-u
\end{array}\right](b-a) \geqslant 0 .
$$

This is the most general definition. We now introduce four special cases in Definitions 2-5.

DEFINITION 2. The matrix $C$ exhibits column dominance of the second type with respect to $u$ (CD2) if $\exists a \geqq 0, b \geqq 0, a^{\prime} b=0$ such that:

$$
C a \leqq C b, \quad u a>u b .
$$

(8) can be rewritten as:

$$
C(b-a) \geqq 0, \quad-u(b-a)>0 .
$$

Definition 3. The matrix $C$ exhibits column dominance of the third type with respect to $u(\mathrm{CD} 3)$ if $\exists a \geqq 0, b \geqq 0, a^{\prime} b=0$ such that:

$$
\mathrm{Ca}<\mathrm{Cb}, \quad u a>u b \text {. }
$$

(11) can be rewritten as:

$$
\left[\begin{array}{c}
C \\
-u
\end{array}\right](b-a)>0
$$

Definition 4. The matrix $C$ exhibits column dominance of the fourth type (CD4) if $\exists a \geqslant 0$ such that:

$$
C a \leqq 0
$$


Definition 5. The matrix $C$ exhibits column dominance of the fifth type (CD5) if $\exists a \geqslant 0$ such that:

$$
\mathrm{Ca}<0 \text {. }
$$

REMARK 1. Corresponding to each of the five types of column dominance is a type of row dominance, denoted by (RD1)-(RD5); the definitions of row dominance are easily obtained by transposition in each of Definitions 1-5.

REMARK 2. The definitions of dominance given above apply to any $m \times n$ matrix $C$, though in our model $m=n .{ }^{4}$ So, $C$ could, for example, be obtained from a single-product industries model with alternative processes in at least one sector.

As a formal example of (CD3), let $C=B-A$, the net output matrix, $u=\ell$, the vector of direct labour inputs, $a$ and $b$ be complementary sub-vectors of an activity vector $x$. Then (CD3) means that it is possible to partition the set of processes into two subsets such that one subset (associated with $b$ ) can produce a larger net output of every commodity than the other subset (associated with $a$ ), using a smaller amount of labour. (CD1) and (CD2) have similar interpretations, with weak inequalities replacing the strong ones as appropriate. (CD4), which can be interpreted as a type of weak column dominance, is a special case of (CD1) with $b=0$; similarly, (CD5), a type of strong column dominance, is a special case of (CD3). Now consider a formal example of (RD3), with $C=B-A$, as above, $v=k$, the vector of sectoral capital values, $a$ and $b$ complementary sub-vectors of a price vector. The matrix $C$ exhibits (RD3) with respect to $v$ if $\exists a \geqq 0, b \geqq 0$, $a^{\prime} b=0$ such that:

or

$$
a C<b C, \quad a v>b v .
$$

$$
(b-a)(C-v)>0 .
$$

(RD3) means that it is possible to partition the set of commodities into two subsets such that one subset (associated with $a$ ) has a lower value of net output than the other (associated with $b$ ) in each process, but that the value of its capital is greater.

As the concepts of dominance are necessarily defined in terms of linear inequalities, the basic mathematical theorems to be employed below in the manipulation of the model are, not surprisingly, theorems of the alternative (see Gale [8] or Mangasarian [10]). We state the required results as Lemmas 1-5; in each case, the alternatives "either" and "or" are to be interpreted strictly, in the sense that only one holds.

\footnotetext{
${ }^{4}$ We rely on the argument advanced by Steedman [15] to support our assumption of equality between numbers of processes and commodities.
} 
LEMMA 1. (Stiemke's Theorem)

$$
\text { Either } z D=0
$$

has a positive solution, or

$$
D x \geqslant 0
$$

has a solution.

LEMMA 2. (Farkas' Theorem)

$$
\text { Either } z D=f
$$

has a non-negative solution, or

$$
\left.\begin{array}{l}
D x \geqq 0 \\
f x<0
\end{array}\right\}
$$

has a solution.

LEMMA 3. (Gordan's Theorem)

$$
\text { Either } z D=0
$$

has a semi-positive solution, or

$$
D x>0
$$

has a solution.

LEMMA 4.

$$
\text { Either } z D \leqq 0
$$

has a semi-positive solution, or

$$
D x>0
$$

has a solution.

\section{LEMMA 5.}

$$
\text { Either } z D<0
$$

has a non-negative solution, or

$$
D x \geqq 0
$$

has a solution.

REMarK 3. To see the connections between the Definitions and the Lemmas, consider first (CD1). $\left[{ }_{-u}^{C}\right]$ in (7) plays the role of $D$ in Lemma $1,(b-a)$ that of $x$. Similarly, each of the other definitions of column dominance is equivalent to one of the alternatives in Lemmas 2-5. Lemmas 1-5 relate to column dominance; by transposition, they can be made to refer to row dominance. 
As an application of one of the definitions of column dominance and the corresponding Lemma, reconsider the third type of column dominance, illustrated above. If (CD3) holds with respect to $\ell$ :

$$
\mathrm{Ca}<\mathrm{Cb}, \quad \ell a>\ell b .
$$

In other words, the processes associated with $b$ are more efficient than those associated with $a$. Then, by Lemma 3:

$$
z\left[\begin{array}{c}
C \\
-\ell
\end{array}\right]=0
$$

does not have a semi-positive solution. With $z \equiv(\tilde{p}, 1),(28)$ can be rewritten as:

$$
\tilde{p} C=\ell
$$

or

$$
\tilde{p} B=\tilde{p} A+\ell .
$$

In effect, the inefficient processes cannot break even at labour-commanded prices, $\tilde{p}$.

Having completed the preliminaries, we can now consider the questions raised in Section 1.1. We begin in Part 2 with the analysis of the quantity system, because underlying any production model must be a productiveness condition analogous to (6). Then in Part 3, we deal with the price system.

\section{The quantity system}

\subsection{Productiveness}

We begin by stating a basic definition for any production model:

Definition 6. The joint production model, summarised in $\{A, \ell: B\}$ is productive if

$$
\exists x \geqslant 0 \text { such that }(B-A) x>0 .
$$

The quantity equations for a uniform growth rate $g$ have been stated in (5). Define:

$$
C(g) \equiv B-(1+g) A .
$$

Then (30) can be rewritten as:

$$
\exists x \geqslant 0 \text { such that } C(0) x>0 .
$$

Immediately, we can derive:

THEOREM 1. The joint production model is productive if and only if $\exists(R D 4)$ at $g=0$. 
Proof. Necessity: From (32) and Lemma 4, it follows that:

$$
z C(0) \leqq 0
$$

does not have a semi-positive solution. So, from Definition 4, 7 (RD4) at $g=0$.

Sufficiency: If $\exists$ (RD4) at $g=0,(33)$ does not have a semi-positive solution, so that (32) follows by Lemma 4.

\subsection{Balanced Growth}

The quantity side of the joint production model has so far been described in terms of equations, as in the single-product industries case. This is satisfactory for the latter, but not for the former. Accordingly, we relax the description to permit weak inequalities in:

DEFINITION 7. There is balanced growth at rate $g$ if $x \geqslant 0$ such that:

$$
B x \geqq(1+g) A x+c \text { or } C(g) x \geqq c .
$$

In this Definition, we take the consumption vector, $c$, as given. To be contrasted with this is the case where $c$ emerges as a residual satisfying (33) when the growth rate is $g$. In other words, we examine the technology not from the point of view of meeting a given $c$ but from that of reproducing itself at rate $g$. So, we have:

DEFINITION 8 . The joint production model is g-productive if $\exists x \geqslant 0$ such that:

$$
C(g) x \geqq 0 \text {. }
$$

From Definition 8 and Lemma 5, we can derive:

THEOREM 2. The joint production model is g-productive if and only if $\exists(R D 5)$ at g.

We can obtain the following result on balanced growth:

THEOREM 3. (i) If $\nexists(R D 4)$ at $\bar{g}$, balanced growth at rate $\bar{g}$ is possible; (ii) if there is balanced growth at rate $\bar{g}, \exists(R D 4)$ for all $g<\bar{g}$.

Proof. (i) If $¥(R D 4)$ at $\bar{g}$ :

$$
a C(\bar{g}) \leqq 0
$$

does not have a semi-positive solution. By Lemma $4, \exists x \geqslant 0$ such that:

$$
C(g) x>0 \text {. }
$$


Let $\bar{x}=\alpha x$, where $\alpha$ is sufficiently large that:

$$
C(\bar{g}) \bar{x} \geqq c .
$$

So, there is the possibility of balanced growth at rate $\bar{g}$.

(ii) If there is balanced growth at rate $\bar{g}$, (38) holds for some $\bar{x} \geqslant 0$. For $g<\bar{g}$ :

$$
\begin{aligned}
C(g) \bar{x} & =[B-(1+g) A] \bar{x} \\
& =[B-(1+\bar{g}) A] \bar{x}+(\bar{g}-g) A \bar{x} \\
& =C(\bar{g}) \bar{x}+(\bar{g}-g) A \bar{x}>c
\end{aligned}
$$

if $A \bar{x}>0$ (i.e. produced means of production are required in each process). So, the existence of balanced growth at rate $\bar{g}$ implies:

$$
\exists \bar{x} \geqslant 0 \text { such that } C(g) \bar{x}>0 \text { for all } g<\bar{g} .
$$

Then by Lemma 4:

$$
a C(g) \leqq 0
$$

does not have a semi-positive solution for $g<\bar{g}$, i.e. $\not$ (RD4) at $g<\bar{g}$.

Two further results follow immediately from this Theorem:

CorollaRY 1. If $\nexists(\mathrm{RD} 4)$ at $\bar{g}$, balanced growth is possible at any rate $g \leqslant \bar{g}$.

CoROLlaRY 2. If there is balanced growth at rate $\bar{g}$ and $c>0, \exists$ (RD4) at $\bar{g}$.

Corollary 1 can be derived using the decomposition illustrated on the right-hand side of (39). The hypotheses of Corollary 2 imply $C(\bar{g}) \bar{x}>0$, for some $\bar{x}>0$, from which the result follows.

\subsection{Fully balanced growth and efficiency}

The state of balanced growth described in (5) can be seen as a special case of Definition 7 . We now give it the name fully balanced growth and investigate its properties.

Definition 9. There is fully balanced growth at rate $g$ if $\exists x \geqslant 0$ such that:

$$
C(g) x=c \text {. }
$$

We can now derive:

THEOREM 4. There is fully balanced growth at rate $g$ if and only if $\exists(R D 3)$ at $g$.

Proof. Necessity: Assume that there is fully balanced growth at rate $g$. Then

$$
C(g) x=c \text { has solution } x \geqslant 0
$$


or

$$
\left(x^{\prime} 1\right)\left[\begin{array}{c}
C(g)^{\prime} \\
-c^{\prime}
\end{array}\right]=0^{\prime} \text { has semi-positive solution. }
$$

Then by Lemma 3:

$$
\left[\begin{array}{c}
C(g)^{\prime} \\
-c^{\prime}
\end{array}\right] q^{\prime}>0
$$

does not have a solution, or

$$
q\left[\begin{array}{c}
C(g) \\
-c
\end{array}\right]>0 \text { does not have a solution, }
$$

or

$$
\left.\begin{array}{l}
q C(g)>0 \\
-q c>0
\end{array}\right\}
$$

does not have a solution. In particular, there is not a vector $q=(b-a)$ which solves (45), where $a$ and $b$ are as in Definition 3. So, $\nexists$ (RD3) at $g$.

Sufficiency. If $\exists(\mathrm{RD} 3)$ at $g, \exists q=(b-a)$ which solves (43), where $a \geqq 0$, $b \geqq 0, a^{\prime} b=0$. To apply Lemma 3 , we need to demonstrate that (45) has no solution. There are two other cases to consider: $q \geqslant 0$ and $q \leqslant 0$. If $q \leqslant 0$ :

$$
q C(g)>0
$$

or

$$
p C(g)<0
$$

where $p=-q \geqslant 0$. (46) implies that $g$ is greater than the largest possible rate of profit, which is a contradiction. So, $\exists q \leqslant 0$ satisfying (45). If $q \geqslant 0$, the second inequality in (45) is clearly contradicted. As (45) does not have a solution, it follows by Lemma 3 that (43) has solution $\left(x^{\prime}, 1\right) \geqslant 0^{\prime}$, i.e. there is balanced growth at rate $g$.

REMARK 4. The structure of this proof will be repeated in others below. That is, in deriving sufficiency, nonexistence of row dominance as above, or column dominance in cases to be considered below, does not permit an immediate appeal to one of the Lemmas; for it must be demonstrated that a set of inequalities does not have any solution, not just no solution with mixed signs. In excluding semi-positive or semi-negative vectors, economic considerations have a role to play. 
Having derived a condition for the existence of fully balanced growth, we now investigate its properties. First of all, we require:

Definition 10. A fully balanced growth path, characterised by the technical coefficients $\{A, \ell: B\}$, rate of growth $g$ and consumption vector $c$ is efficient if there does not exist another balanced path satisfying the same consumption vector with $\tilde{g} \geqslant g$, such that a larger amount of labour is not employed. That is, $\nexists$ $\tilde{x}$ such that:

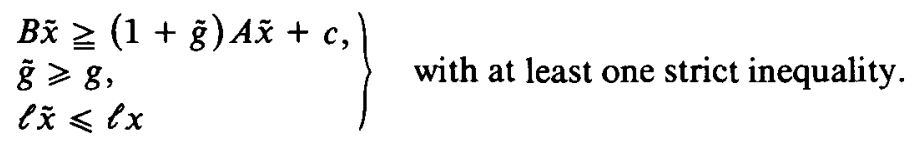

This definition, familiar in the economics literature on growth theory, can be simplified somewhat. Given $\tilde{x}$, define $\tilde{g}_{\imath} \equiv g_{\imath}(x)$ by:

$$
B_{i} \tilde{x} \equiv\left(1+\tilde{g}_{i}\right) A_{i} \tilde{x}+c_{i},
$$

where $A_{i}$ denotes the $i$-th row of $A, B$, the $i$-th row of $B$. From (48) and the first two inequalities of (47), it is clear that $\tilde{g}_{1} \geqslant g$ for all $i$. Combining these with the third inequality, we have instead of (47):

$$
\left.\begin{array}{l}
\tilde{g}_{\imath} \geqslant g, \quad i=1, \ldots, n \\
\ell \Delta x \leqslant 0
\end{array}\right\} \quad \text { with at least one strict inequality, }
$$

where $\Delta x=\tilde{x}-x$. We can now derive:

THEOREM 5. Fully balanced growth at rate $g$ is efficient if and only if $\exists(C D 1)$ at g.

Proof. First of all, we transform (49) into a linear inequality involving $C$ and $\ell$. From (48):

$$
B_{i} \tilde{x}=(1+g) A_{i} \tilde{x}+\left(\tilde{g}_{i}-g\right) A_{i} \tilde{x}+c_{i} .
$$

The $i$-th component of (42) is:

$$
B_{i} x=(1+g) A_{i} x+c_{i} .
$$

Subtracting (51) from (50):

$$
B_{i} \Delta x=(1+g) A_{i} \Delta x+\left(\tilde{g}_{i}-g\right) A_{i} \tilde{x}
$$

or

$$
C_{i}(g) \Delta x=\left(\tilde{g}_{i}-g\right) A_{i} \tilde{x},
$$

where $C_{i}(g)$ is the $i$-th row of $G(g)$. Stacking (50) for $i=1, \ldots, n$, we obtain:

$$
C(g) \Delta x=G A \tilde{x},
$$


where $G=\left[\left(\tilde{g}_{i}-g\right) \delta_{i j}\right]$. Using (52) and (53), the first inequality in (49) can be rewritten as:

$$
C(g) \Delta x \geqq 0 .
$$

Hence, (49) can be rewritten as:

$$
\left.\begin{array}{l}
C(g) \Delta x \geqq 0, \\
-\ell \Delta x \geqslant 0
\end{array}\right\} \quad \text { with at least one strict inequality }
$$

or

$$
\left[\begin{array}{c}
G(g) \\
-\ell
\end{array}\right] \Delta x \geqslant 0
$$

This is the desired inequality.

Necessity: If balanced growth is efficient, (55) does not have a solution: in particular, it does not have a solution $\Delta x$ of mixed signs as in Definition 1 . So 7 (CD1) at $g$.

Sufficiency: Assume $\nexists(\mathrm{CD} 1)$ at $g$. Then (55) does not have a solution with mixed signs. Consider then $\Delta x \geqslant 0$ and $\Delta x \leqslant 0 . \Delta x \geqslant 0$ is impossible as $-\ell \Delta x \geqslant 0$ in (55) with $\ell>0$. This leaves $\Delta x \leqslant 0$. Consider:

$$
C(g) \Delta x \geqq 0
$$

or

$$
C(g) y \leqq 0,
$$

with $y=-\Delta x \geqslant 0$. (56) implies that $g$ is greater than or equal to the maximum uniform rate of growth with $c \geqslant 0$. This is a contradiction. So, (55) does not have a solution, which implies that fully balanced growth is efficient.

Remark 5. The existence results, Theorems $1-4$, are in terms of row dominance conditions.

This completes the discussion of the quantity equations. In Part 3, we consider the price equations.

\section{The price equations}

\subsection{Price Systems}

In our discussion of the quantity side, we distinguished between balanced and fully balanced growth, the latter characterised by a uniform rate of growth. We draw a similar distinction in discussing the price equations between a price system and prices of production, the latter defined in terms of a uniform rate of growth. 
Definition 11. A price system for the joint production model is a semi-positive vector $p$ such that:

$$
p B^{\prime}=\left(1+r_{j}\right) p A^{\prime}+w_{j} \ell, j=1, \ldots, n .
$$

A price system need not imply a uniform rate of either wages of profits, as can be seen in the next two Theorems:

THEOREM 6. There is a price system with uniform rate of profit $r$, but not necessarily uniform wage rate, if and only if $\exists(C D 4)$ at $r$.

Proof. Necessity: Assume that there exists a price system with uniform rate of profit $r$. (57) can be rewritten as:

$$
p B=(1+r) p A+\ell W,
$$

where $W=\left[w_{i} \delta_{i j}\right]$, or

$$
p C(r)=\ell W>0
$$

or

$$
C(r)^{\prime} p^{\prime}>0
$$

has solution $p \geqslant 0$. By Lemma $4, \exists a \geqslant 0$ such that:

$$
a^{\prime} C(r)^{\prime} \leqq 0
$$

or

$$
C(r) a \leqq 0,
$$

i.e. $\nexists(\mathrm{CD} 4)$ at $r$.

Sufficiency: Reverse the previous argument.

Alternatively, it is possible to have a uniform wage rate, as in:

THEOREM 7. There is a price system with uniform wage rate, but not necessarily a uniform rate of profit, if and only if $\exists(C D 4)$ at $r$; in which case, the average rate of profit is equal to $r$.

Proof. Sufficiency: Assume $\nexists(C D 4)$ at $r$. Then by Definition 4 and Lemma 4, $\exists p \geqslant 0$ such that:

$$
C(r)^{\prime} p^{\prime}>0
$$

or

$$
p C(r)>0 .
$$

$p C(r)$ is not necessarily proportional to $\ell$. If it is not, define:

$$
\tilde{w}=p C(r) x / \ell x .
$$


$\tilde{w}$ plays the role of a uniform wage rate. With the price vector $p$ from (61) and the uniform wage, $\tilde{w}$, from (62), define the sectoral rates of profit by:

$$
p\left[B^{J}-\left(1+r_{j}\right) A^{j}\right]=\tilde{w} \ell_{j}
$$

or

$$
r_{j}=\left[\bar{p}\left(B^{j}-A^{l}\right)-\tilde{w} i_{j}\right] / \dot{p} A^{j} .
$$

The average rate of profit for the economy as a whole is:

$$
\bar{r}=[p(B-A) x-\tilde{w} \ell x] / p A x
$$

where $x$ is an activity vector. From (62) and (64), it follows that $\bar{r}=r$, as required.

Necessity: Suppose that there is a price system with a uniform wage rate and an average rate of profit equal to $r$. Reversing the above argument yields (61), whence, by Lemma $4, \exists$ (CD4) at $r$.

\subsection{Prices of production}

In this Section, we deal with prices of production, which constitute a special case of price systems, as mentioned above.

Definition 12. Prices of production, $p \geqslant 0$, solve:

$$
p B=(1+r) p A+w \ell \text {. }
$$

As the wage is uniform, it is possible to divide through (65) by $w>0$ to obtain:

$$
\tilde{p} B=(1+r) \tilde{p} A+\ell,
$$

where $\tilde{p} \equiv p / w$ is the vector of labour-commanded prices. From (42) and (66), there is a duality between fully balanced growth and prices of production. It is thus to be expected that a dominance condition will (not) hold for prices of production analogous to the dominance condition which does (not) hold for fully balanced growth.

THEOREM 8. Prices of production exist at $r$ if and only if $¥(C D 3)$ at $r$.

Proof. See Filippini and Filippini [6].

Insofar as labour values are prices of production evaluated at zero rate of profit, this result follows:

Corollary 3. Labour values exist if and only if $\nexists(\mathrm{CD} 3)$ at $r=0$. 
REMARK 6. For an $n$-commodity, $n$-process, single-product industries model, labour values can be derived from prices of production by putting the rate of profit equal to zero. For $m$-commodity, $n$-process single-product industries models $(n>m)$ and $n$-commodity, $n$-process, multiple-product industries models, this procedure has been questioned by Toker [16].

Remark 7. Prior to Theorem 8, we drew attention to the duality between fully balanced growth and prices of production. Now, in Remark 5, we pointed to the connection between existence results on the quantity system and non-existence of a row dominance condition. Similarly, it can be seen from Theorems 6-8 that existence results on the price system are connected with non-existence of a column dominance condition. As efficiency of fully balanced growth is derived in Theorem 5 in terms of a column dominance condition, it might be supposed that there is an associated set of prices of production-this is confirmed in:

COROLlaRY 4. Efficient fully balanced growth at rate $g$ is supported by prices of production.

Proof. By Theorem 5, if there is efficient fully balanced growth at rate $g, 7$ (CD1) at $g$. Then from Lemma 1:

$$
z\left[\begin{array}{c}
C(g) \\
-\ell
\end{array}\right]=0
$$

has a positive solution $z=(p, w)$. That is:

$$
p C(g)=w \ell
$$

or

$$
p B=(1+g) p A+w \ell .
$$

\subsection{Price variations and conflict in distribution}

In single-product industries models, labour-commanded prices are increasing functions of the rate of profit. In the first result of this Section, we present a necessary and sufficient condition for this to occur in our joint production model.

THEOREM 9. Labour-commanded prices are increasing functions of $r$ if and only if $\exists(C D 1)$ at $r$.

Proof. See Filippini and Filippini [6]. 
Remark 8. Theorem 9 can be applied to an $n$-commodity, $n$-process, singleproduct industries model to derive the result mentioned at the beginning of this Section. This follows because the (CD1) condition is not satisfied at any feasible value of $r$.

COROllary 5. The $w-r$ curve, in terms of any numeraire, is downward-sloping at $r$ and and only if $\exists(C D 1)$ at $r$.

Proof. As $\tilde{p}_{i}=1 / \tilde{w}_{t}=w / p_{i}$, it follows that $d \tilde{p}_{i} / d r=-\left(d \tilde{w}_{i} / d r\right) / \tilde{w}_{i}^{2}$. As the two derivatives have opposite signs, the result follows from Theorem 9.

This Corollary can be interpreted in terms of distributional conflict over the share of the national product between workers and capitalists. A more general definition would be as follows:

Definimion 13. There is distributional conflict at $r^{*}$ if there does not exist a price system $p$ such that:

$$
\left.\begin{array}{l}
r_{j} \equiv r_{j}(p) \geqslant r^{*} \text { for all } j \\
p y \leqslant w
\end{array}\right\} \text { with at least one strict inequality, }
$$

where $y$ is a fixed wage basket. That is, there is not a price system yielding a rate of profit on each process greater than or equal to $r^{*}$, with the wage basket costing at most $w$. In other words, it is impossible to find a price system that would be preferred by workers as a class or by capitalists in each industry.

THEOREM 10. There is distributional conflict at $r^{*}$ if and only if $\exists(R D 1)$ at $r^{*}$.

Proof. First of all, we manipulate (69) to obtain a linear inequality in $C$ and $y$. If there are non-uniform rates of profit:

$$
p B^{\prime}=\left(1+r_{j}\right) p A^{J}+w \ell_{j}
$$

or

$$
\tilde{p}\left[B^{j}-\left(1+r_{j}\right) A^{j}\right]=\ell_{j} .
$$

At $r=r^{*}$ :

$$
\tilde{p}^{*}\left[B^{J}-\left(1+r^{*}\right) A^{j}\right]=\ell_{j}
$$

Rewrite (70) as:

$$
\tilde{p}\left[B^{j}-\left(1+r^{*}\right) A^{j}\right]=\ell_{j}+\left(r_{j}-r^{*}\right) \tilde{p} A^{j} .
$$

Subtracting (71) from (72), we have, with $\Delta \tilde{p}=\tilde{p}-\tilde{p}^{*}$ :

$$
\Delta \tilde{p}\left[B^{j}-\left(1+r^{*}\right) A^{J}\right]=\left(r_{j}-r^{*}\right) \tilde{p} A^{j}
$$


or

$$
\Delta \tilde{p} C^{J}\left(r^{*}\right)=\left(r_{j}-r^{*}\right) \tilde{p} A^{J}
$$

As $r_{j} \geqslant r^{*}$ is equivalent to $\Delta \tilde{p} C^{J}\left(r^{*}\right) \geqslant 0$, it follows that the first inequality in (69) can be rewritten as:

$$
\Delta \tilde{p} C\left(r^{*}\right) \geqq 0 .
$$

As $w=p^{*} y$, the second inequality in (69) can be rewritten as:

$$
\Delta \tilde{p} \leqslant 0 .
$$

Combine (74) and (75) to yield:

$$
\Delta \tilde{p}\left[C\left(r^{*}\right)-y\right] \leqslant 0,
$$

which is the desired inequality.

Necessity: If there is distributional conflict at $r^{*}$, it follows from above that (76) does not have a solution, in particular a mixed solution $\Delta \tilde{p}=(b-a)$ as in Definition 1. So $\nexists$ (RD1) at $r^{*}$.

Sufficiency: Assume $\nexists$ (RD1) at $r^{*}$. Then (76) does not have a mixed solution $\Delta \tilde{p}=(b-a)$. It remains to consider $\Delta \tilde{p} \geqslant 0$ and $\Delta \tilde{p} \leqslant 0 . \Delta \tilde{p} \geqslant 0$ is impossible as $\Delta \tilde{p} y \leqslant 0$. So finally consider $\Delta \tilde{p} \leqslant 0$. Then

$$
\Delta \tilde{p} C\left(r^{*}\right) \geqq 0
$$

or

$$
-\Delta \tilde{p} C\left(r^{*}\right) \leqq 0,-\Delta \tilde{p} \geqslant 0 .
$$

This implies that $r^{*}$ is greater than or equal to the maximum uniform rate of profit, which is a contradiction. So (76) does not have a solution. Hence there is distributional conflict at $r^{*}$.

REMARK 9. Further to Remarks 5 and 7 and Corollary 4, we see that, if there is distributional conflict, there is the possibility of fully balanced growth at rate $r^{*}$ (and vice versa). This is established in:

Corollary 6 . There is the possibility of balanced growth at rate $r^{*}$ if and only if there is distributional conflict at $r^{*}$.

Proof. From Lemma 1, if (76) does not have a solution:

$$
\left[C\left(r^{*}\right)-y\right]\left[\begin{array}{l}
x \\
1
\end{array}\right]=0
$$


has a positive solution. So:

$$
C\left(r^{*}\right) x=y, x>0 .
$$

This completes the analysis of the price system. In Part 4 , we consider a numerical example.

\section{An example}

We apply the results of Parts 2 and 3 to the following example, introduced by Steedman [14]:

$$
A=\left[\begin{array}{cc}
5 & 0 \\
0 & 10
\end{array}\right], \quad B=\left[\begin{array}{cc}
6 & 3 \\
1 & 12
\end{array}\right], \quad C(r)=\left[\begin{array}{cc}
1-5 r & 3 \\
1 & 2-10 r
\end{array}\right], \quad l=(1,1) .
$$

Clearly, the system is productive,i.e. $\exists(\mathrm{RD} 4)$ at $r=0$. The system is $g$-productive for all $g<g_{\max }=(2+\sqrt{6}) / 10$, which is obtained as the smallest positive solution to:

$$
\operatorname{det} C(g)=0 .
$$

Fully balanced growth at rate $g$ obtains if and only if $\exists$ (RD3) at $g$; this means, by Lemma 3 , that

$$
C(g) x=c
$$

has solution $x \geqslant 0$. From (79) and (81), we have:

$$
x=\left[\begin{array}{c}
\left(2 c_{1}-3 c_{2}\right)-10 c_{1} g \\
\left(c_{2}-c_{1}\right)-5 c_{2} g
\end{array}\right] / \operatorname{det} C(g)
$$

where det $C(g)=50 g^{2}-20 g-1$. We see that (82) places restrictions on $c$. e.g. if $g=0, c$ must satisfy:

$$
2 c_{1} / 3 \leqslant c_{2} \leqslant c_{1} .
$$

On the other hand, if $c=e^{1}$, the first unit vector of order two, it follows from (82) that:

$$
x^{1}=C(g)^{-1} e^{1}=\left[\begin{array}{c}
(2-10 g) \\
-1
\end{array}\right] / \operatorname{det} C(g)>0
$$

if $g \in\left(1 / 5, g_{\max }\right)$. Similarly, if $c=e^{2}$ :

$$
x^{2}=C(g)^{-1} e^{2}=\left[\begin{array}{c}
-3 \\
(1-5 g)
\end{array}\right] / \operatorname{det} C(g)>0
$$

if $g \in\left(1 / 5, g_{\max }\right)$. But if $c=\underline{1}=e^{1}+e^{2}$ :

$$
x=C(g)^{-1} \underline{1}=\left[\begin{array}{c}
(-1-10 g) \\
-5 g
\end{array}\right] / \operatorname{det} C(g)>0
$$


if $g \in\left(0, g_{\max }\right)$. This demonstrates that the range of values of $g$ over which balanced growth exists depends on the specification of $c$.

Fully balanced growth at rate $g$ is efficient if and only if $\exists$ (CD1) at $g$. From Lemma 1, this is equivalent to:

$$
\left[\begin{array}{ll}
z & 1
\end{array}\right]\left[\begin{array}{c}
C(g) \\
-\ell
\end{array}\right]=0
$$

having a positive solution. From (79) and (84), we see that this obtains if and only if $g \in\left(1 / 10, g_{\max }\right)$.

Turning now to the price side, we see, by applying Theorems 6 and 7, that a price system exists for all $r \in\left[0, r_{\max }\right]$, where $r_{\max }$ solves (80). From Theorem 8 and Lemma 3, prices of production are given by:

$$
\begin{gathered}
p=l C(r)^{-1}=(1-10 r,-2-5 r) / \operatorname{det} C(r) \\
\geqslant 0 \text { if } r \in\left(1 / 10, r_{\max }\right) .
\end{gathered}
$$

So, prices of production do not exist at $r=0$, as demonstrated by Steedman [14].

From Theorem 9, labour-comanded prices are increasing at $r$ if and only if $\exists$ (CD1) at $r$, which from Theorem 5 occurs for $r \in\left(1 / 10, r_{\max }\right)$. Distributional conflict, in the sense of Theorem 10, arises if and only if $\exists$ (RD1) at $r$. From Lemma 1, this means that:

$$
[C(r)-y]\left[\begin{array}{l}
x \\
1
\end{array}\right]=0
$$

has a positive solution:

$$
x=C(r)^{-1} y>0 .
$$

(86) depends on the specification of $y$. If $y=e^{1}$ or $e^{2}$, (86) is satisfied when $r \in\left(1 / 5, r_{\max }\right)$. If $y=\underline{1},(86)$ is satisfied when $r \in\left(0, r_{\max }\right)$.

\section{Conclusion}

In Parts 2 and 3, we have exhibited a duality between price and quantity systems. Existence results on the former are connected with column dominance while those for the latter are connected with row dominance. On the other hand, properties of the former (such as distributional conflict or prices of production being increasing functions of $r$ ) are related to row dominance, while those for the latter (such as efficiency of balanced growth) are related to column dominance. In the analysis, we have employed the first, third, fourth and fifth dominance concepts. This is not to imply redundance of the second concept, for it can be used to derive a weaker version of Theorem 9 (i.e. labour-commanded prices are non-decreasing functions of $r$ if and only if $\nexists(\mathrm{CD} 2)$ at $r$ ). 


\section{Acknowledgements}

I am grateful to the referees for their advice: the usual disclaimer applies. I would also like to thank the Department of Economics, Queen Mary College, University of London, especially Mr. R. J. Allard, for the provision of facilities.

\section{References}

[1] A. Berman and R. J. Plemmons, Nonnegative matrices in the mathematical sciences (Academic Press, London, 1979).

[2] E. Burmeister and A. R. Dobell, Mathematical theories of economic growth (Collier-Macmillan, London, 1970).

[3] G. Dumenil and D. Levy, "Valeurs et prix de production", Revue Economique 33 (1982), 30-70.

[4] C. Filippini, "Positivita dei prezzi e produzione congiunta", Giornale degli Economisti e Annali di Economia 36 (1977), 91-99.

[5] L. Filippini and C. Filippini, "La relazione tra saggio di salario a saggio di profitto in produzione congiunta", Rivista Internazionale di Scienze Sociale 87 (1979), 425-441.

[6] L. Filippini and C. Filippini, "Two theorems on joint production", Economic Journal 92 (1982), 386-390.

[7] Y. Fujimori, Modern analysis of value theory (Springer-Verlag, Berlin, 1983).

[8] D. Gale, The theory of linear economic models (McGraw-Hill, London, 1960).

[9] D.Levy, "La formalisme unificateur du surclassement", in La Production Jointe (ed. C. Bidard), (Economica, Paris, 1985).

[10] O. L. Mangasarian, Nonlinear programming (McGrw-Hill, London, 1969).

[11] L. L. Pasinetti, "The notion of vertical integration in economic analysis", Metroeconomica 25 (1973), 1-29.

[12] L. L. Pasinetti, Lectures on the theory of production (Macmillan, London, 1977).

[13] P. Sraffa, Production of commodities by means of commodities (Cambridge University Press, Cambridge, 1960).

[14] I. Steedman, "Positive profits with negative surplus value", Economic Journal 85 (1975), 114-123.

[15] I. Steedman, "Positive profits with negative surplus value: a reply to Wolfstetter", Economic Journal 86 (1976), 873-876.

[16] M. A. Toker, "A note on 'negative' quantities of embodied labour", Economic Journal 94 (1984), 149-154.

[17] J. E. Woods, Mathematical economics (Longmans, London, 1978). 